\title{
Metallic Magnetic Nanocrystals - Shapes, Self-assembly and Phase Transformation
}

\author{
Zhong Lin (ZL) Wang*, Zurong Dai* and Shouheng Sun**
}

* Center for Nanoscience and Nanotechnology, and School of Materials Science and Engineering, Georgia Institute of Technology, Atlanta, GA 30332-0245; *e-mail: zhong.wang@mse.gatech.edu ** IBM T. J. Watson Research Center, Yorktown Heights, New York 10598

Controlled assembly of magnetic nanocrystals (NCs) has been a key issue in fabricating functional nanodevices. Extensive investigations in $\mathrm{NC}$ assemblies have revealed that the symmetry of the observed superlattices is influenced by the NC size, NC shape and relative dimensions of the NC core and the organic capping. Our study has been focused on investigating the roles played by NC shape in self-assembly and the mechanism by which defects are created [1]. The key challenges in this self-assembly method are to control the defects of the self -assembly to ensure an ordered structure, and to prepare an assembly with uniform thic kness and large lateral dimension. For the $11 \mathrm{~nm}$ Co NCs and their superlattice assembly (Fig. 1), the Co NCs have anisotropic polyhedral shapes (Fig. 2) [2]. The self -assembly of the NCs and the defect structures in the NC arrays are governed by the anisot ropic shape. The self-assembly of nanocrystals with faceted shape is dominated by a surface-to-surface contact. This simple geometrical matching is the root for creating defects in the self-assembly. The structural transformation of shape-controlled nanocrystals has been studied by transmission electron microscopy.

Our second study is about the FePt nanocrystals, which have been demonstrated as a potential candidate for high-density magnetic transitions at room temperature [3]. Depending on the Fe to $\mathrm{Pt}$ elemental ratio, the Fe-Pt alloys can display chemically disordered face centered cubic (fcc) phase $(\mathrm{A} 1, \mathrm{Fm} \overline{3} \mathrm{~m})$ or chemically ordered phases. High-resolution transmission electron microscopy (HRTEM) studies show that $\mathrm{A} 1$ to $\mathrm{L} 1_{0}$ phase transformation occurs at $530{ }^{\circ} \mathrm{C}[4,5]$. The multilayered nanocrystal assemblies coalesce to form larger grains at $600{ }^{\circ} \mathrm{C}$. The coalescent temperature of the nanocrystal monolayer assembly depends on the substrate used (Fig. 3). On $\mathrm{SiO}_{2}$ surface, the FePt nanocrystal monolayer can stand up to $700{ }^{\circ} \mathrm{C}$ without any obvious aggregation. The coalesced nanocrystals show dominant $\{111\}$ twin defect inside, while their surface and coalescent grain boundary consist of both $\{111\}$ and (001) facets [6].

References

[1] Z.L. Wang (ed.) Characterization of Nanophase Materials (Wiley-VCH, 2000);

Z.L. Wang, Adv. Mater. 10 (1998) 13; Z.L. Wang, J. Phys. Chem. B, 104 (2000) 1153;

J.S. Yin and Z.L. Wang, Phys. Rev. Lett., 79 (1997) 2570.

[2] Z.L. Wang, Z.R. Dai and S. Sun, Adv. Mater., 12 (2000) 1944.

[3] S. Sun, C. B. Murray, D. Weller, L. Folks, A. Moser,Science, 287 (2000) 1989.

[4] Z. R. Dai, Z. L. Wang, and Shouheng Sun "Phase Transformation, Coalescence and Twinning of Monodisperse FePt Nanocrystals", Nanoletters. 1 (2001) 443-447.

[5] Z.R. Dai, Z. L. Wang, and S.H. Sun "Shapes, Multiply Twins and Surface Structures of Monodispersive FePt Magnetic Nanocrystals", Surface Science, submitted (2001).

[6] Research was supported by the NSF (DMR-9733160) and DARPA (DAAD19-01-1-0546). 

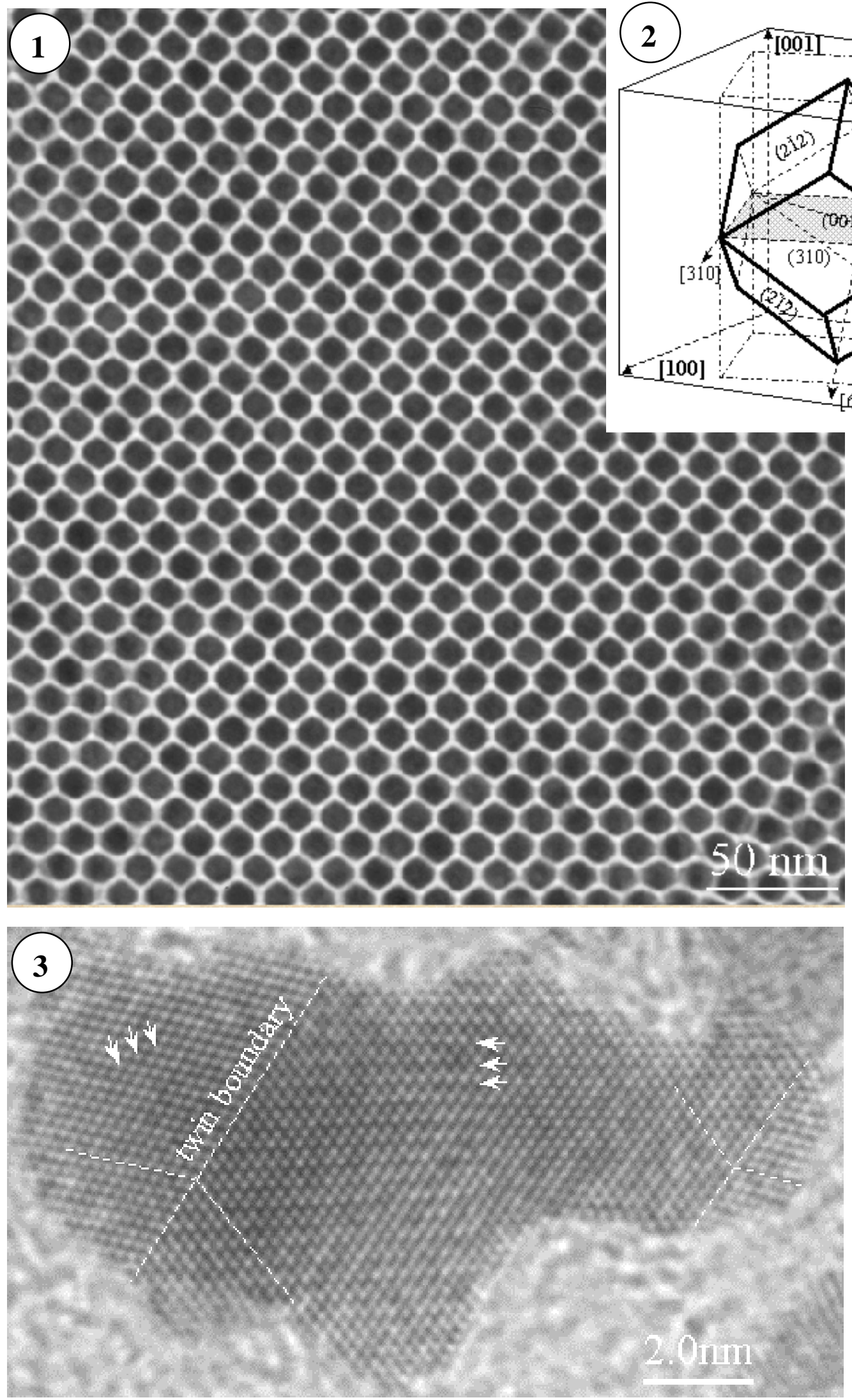

Fig. 1 Selfassembly of 11 $\mathrm{nm} \mathrm{Co}$ nanocrystals forming an ordered structure with translation symmetry but no long-range orientation symmetry.

Fig. 2 The shape of the $11 \mathrm{~nm} \mathrm{Co}$ particle $(\varepsilon-\mathrm{Co}$ phase with $\beta-\mathrm{Mn}$ structure).

Fig. 3 Coalescing and phase transformation of $\mathrm{FePt}$ nanocrystals. The twin is formed due to the coalescing of two particles, and the ordered $\mathrm{Fe}$ and $\mathrm{Pt}$ stacking is indicated by arrowheads. 